\title{
Fracture Healing via Periosteal Callus Formation Requires Macrophages for Both Initiation and Progression of Early Endochondral Ossification
}

\author{
Liza J. Raggatt, ${ }^{* \dagger}$ Martin E. Wullschleger, ${ }^{\ddagger \S}$ Kylie A. Alexander, ${ }^{\dagger}$ Andy C.K. Wu, ${ }^{\dagger}$ Susan M. Millard, ${ }^{*}$ Simranpreet Kaur, ${ }^{* \dagger}$
} Michelle L. Maugham, ${ }^{*}$ Laura S. Gregory, ${ }^{\top}$ Roland Steck, ${ }^{\top}$ and Allison R. Pettit**

\begin{abstract}
From the Bone and Immunology Laboratory, * Mater Research Institute-UQ, Translational Research Institute, The University of Queensland, Woolloongabba, Queensland; the UQ-Centre for Clinical Research ${ }^{\dagger}$ and School of Medicine, ${ }^{\S}$ Faculty of Health Sciences, The University of Queensland, Herston, Queensland; the Trauma Service ${ }^{\ddagger}$ Royal Brisbane and Women’s Hospital, Herston, Queensland; and the Institute of Health and Biomedical Innovation, "Queensland University of Technology, Kelvin Grove, Queensland, Australia
\end{abstract}

Accepted for publication August 21, 2014.

Address correspondence to Allison R. Pettit, Ph.D., Mater Research-UQ, Translational Research Institute, Level 4 , Room 4015, 37 Kent St, Woolloongabba, QLD 4102, Australia. E-mail: allison. pettit@mater.uq.edu.au.

\begin{abstract}
The distribution, phenotype, and requirement of macrophages for fracture-associated inflammation and/or early anabolic progression during endochondral callus formation were investigated. A murine femoral fracture model [internally fixed using a flexible plate (MouseFix)] was used to facilitate reproducible fracture reduction. IHC demonstrated that inflammatory macrophages $\left(\mathrm{F} 4 / 80^{+} \mathrm{Mac}-2^{+}\right)$were localized with initiating chondrification centers and persisted within granulation tissue at the expanding soft callus front. They were also associated with key events during soft-to-hard callus transition. Resident macrophages (F4/ $\left.80^{+} \mathrm{Mac}-2^{\text {neg }}\right)$, including osteal macrophages, predominated in the maturing hard callus. Macrophage Fasinduced apoptosis transgenic mice were used to induce macrophage depletion in vivo in the femoral fracture model. Callus formation was completely abolished when macrophage depletion was initiated at the time of surgery and was significantly reduced when depletion was delayed to coincide with initiation of early anabolic phase. Treatment initiating 5 days after fracture with the pro-macrophage cytokine colony stimulating factor-1 significantly enhanced soft callus formation. The data support that inflammatory macrophages were required for initiation of fracture repair, whereas both inflammatory and resident macrophages promoted anabolic mechanisms during endochondral callus formation. Overall, macrophages make substantive and prolonged contributions to fracture healing and can be targeted as a therapeutic approach for enhancing repair mechanisms. Thus, macrophages represent a viable target for the development of pro-anabolic fracture treatments with a potentially broad therapeutic window. (Am J Pathol 2014, 184: 3192-3204; http://dx.doi.org/10.1016/j.ajpath.2014.08.017)
\end{abstract}

Bone fracture resulting from disease- and age-related bone fragility or traumatic injury is common and costly. ${ }^{1-3}$ There are no routine first-line therapies that accelerate healthy bone repair and/or prevent bone nonunion. Development of such therapies would complement existing orthopedic approaches and reduce the unacceptably high burden of fracture. ${ }^{2}$ Fracture healing is a complex process that requires the coordinated interplay of many cell types, growth factors, extracellular matrix components, and mechanobiological input. ${ }^{4}$ Healing of nonrigidly stabilized fractures occurs via generation of a periosteal callus that bridges the fracture site. In this setting, the anabolic mechanism is predominantly endochondral ossification. ${ }^{5}$ Periosteal endochondral callus formation progresses via four sequential and interdependent phases: inflammation leading to granulation tissue formation, early anabolism (soft cartilaginous callus formation), late anabolism (hard bony callus formation), and remodeling to

\footnotetext{
Supported by National Health and Medical Research Council (NHMRC) project grant 631484 (A.R.P. and L.J.R.), NHMRC Dora Lush Biomedical Scholarship 409914 (K.A.A.), NHMRC Career Development Award 519744 (A.R.P.), and the Mater Foundation.

Disclosures: None declared.

Current address of K.A.A., Clive Berghoffer Cancer Research Centre, Queensland Institute for Medical Research, Herston, Queensland; of A.C.K.W., Mater Research Institute-UQ, Translational Research Institute, Woolloongabba, Queensland, Australia.
} 
reinstate the original bone architecture and mechanical strength. ${ }^{5}$ Although there can be temporal overlap between progressive phases, the cellular processes are generally spatially segregated in a site-specific manner within the forming callus.

The importance of appropriately controlled inflammation during the initiation of fracture repair is established. ${ }^{6,7}$ Initiation of fracture healing is characterized by infiltration of inflammatory cells to the fracture site, which combat infection and clear debris and dead cells. These inflammatory cells also secrete a variety of cytokines and growth factors that are important for directing neovascularization ${ }^{8}$ and cell recruitment. ${ }^{9-11}$ We and others have clearly demonstrated that macrophages are present during the inflammatory phase of fracture healing in humans ${ }^{12,13}$ and animals. ${ }^{14-16}$ Although macrophages have been implicated as important contributors to the inflammatory events initiating bone repair, ${ }^{9-11}$ their requirement for these events has not been definitively established.

There has been limited consideration of macrophage contributions beyond the initial inflammatory phase despite data from animal models describing the presence of macrophages within repair-associated tissues during the early anabolic phase. ${ }^{14,17,18}$ In addition, macrophages persisted in human fracture-associated tissues collected at a range of time points after fracture and most notably were associated with areas of bone formation. ${ }^{12}$ There is a lack of definitive evidence supporting direct macrophage contributions to anabolic processes during endochondral callus formation. Altered endochondral callus formation has been reported as a result of manipulation of macrophage migration and/or macrophage-expressed molecules, but the experimental designs did not specifically target these molecules during the anabolic phase. ${ }^{15,17,19,20}$ Therefore, altered inflammatory phase progression/resolution with knock-on consequences on later repair phases cannot be ruled out as the primary mechanism leading to the compromised fracture repair in these studies.

Macrophages can be broadly categorized into two overarching populations, resident and inflammatory macrophages. Resident macrophages are present throughout life in virtually all tissues, making vital contributions to tissue development, homeostasis, and repair. Resident macrophage populations adapt tissue-specific phenotypes and functional abilities. ${ }^{21}$ Dogma suggests that resident macrophages are replenished from a circulating monocyte pool; however, more recent data indicate potential alternative seeding and lifelong maintenance mechanisms. ${ }^{22-24}$ Resident macrophages can be derived from primordial hematopoietic progenitors, ${ }^{22}$ can be resistant to lethal irradiation, ${ }^{23,24}$ and are able to locally self-replenish without ongoing input from bone marrow hematopoiesis. ${ }^{22-24}$ In contrast, recruited inflammatory macrophages are derived from blood monocytes and rapidly infiltrate tissues compromised by injury, abnormal function, and/or infection. ${ }^{25}$ These macrophages are generally short lived $^{26}$ and, depending on the environmental cues encountered within the infiltrated tissue, are polarized toward a specific inflammatory profile. Macrophage activation occurs across a spectrum, with the two extremes described as M1 (classic activation, critical in destroying foreign organisms and fighting infection) and M2 (alternative activation, implicated in wound healing, tissue repair, inflammation resolution, debris scavenging, and angiogenesis). ${ }^{27}$

Bone tissues contain a resident macrophage population (osteomacs) that provides pro-anabolic support to osteoblasts. ${ }^{28}$ This discovery exposed a prominent and previously unappreciated role for macrophages in bone biology with obvious implications for fracture repair. Osteomacs were required for maintenance of mature osteoblasts in vivo, and depletion of bone marrow macrophages, including osteomacs, was associated with significant reductions in histomorphometric parameters of bone formation during bone modeling. ${ }^{28,29}$ There is a growing body of evidence supporting macrophage influence on pro-anabolic mechanisms. It was recently demonstrated that macrophages are required for skeletal homeostasis and anabolic actions of parathyroid hormone. ${ }^{30}$ Human inflammatory monocytes/macrophages have also been shown to influence bone biology, including via production of oncostatin $\mathrm{M}$, which was demonstrated to be pro-anabolic both in vitro and in vivo. ${ }^{31,32}$ Another study using collagen-based scaffolds in a cranial defect model observed that inflammatory macrophages were associated with areas of anabolic response, ${ }^{33}$ and they have been recently implicated in biomaterial-induced osteogenesis. ${ }^{34}$ Finally, we have shown that osteomacs were critical participants during bone healing via intramembranous ossification in a mouse tibial injury model. ${ }^{14}$ The specific macrophage subtype associated with each phase of fracture repair during endochondral callus formation has not been determined.

Herein, we investigated the distribution of inflammatory versus resident macrophages, including osteomacs, during endochondral ossification using a nonrigid internally plated femoral fracture mouse model (MouseFix; RISystem, Davos Platz, Switzerland). ${ }^{35-37}$ To delineate between phase-specific functional contributions of macrophages to fracture healing, we used an inducible model of macrophage depletion [macrophage Fas-induced apoptosis (Mafia) transgenic mice ${ }^{38}$. We used appropriately timed treatment regimens to achieve macrophage depletion either initiating during fracture-associated early inflammation or coinciding with anabolic progression of bone repair. We also used appropriately timed delivery of colony-stimulating factor-1 (CSF-1), which is required for proliferation and differentiation of mononuclear phagocyte progenitor cells to monocytes, macrophages, and osteoclasts, ${ }^{39,40}$ to enhance macrophages specifically during the early anabolic phase of repair.

\section{Materials and Methods}

Animals

Animal experiments were undertaken as dictated by the Australian code for the care and use of animals for scientific 
purposes. Mafia transgenic mice ${ }^{38}$ and C57Bl/6 control animals were obtained from institutional or commercial (Animal Resources Center, Canning Vale, WA) breeding colonies. Mafia mice, which express a ligand-inducible, Fas-dependent suicide receptor under the control of the myeloid-specific Csflr promoter, ${ }^{41}$ were a generous gift from Prof. Donald Cohen (University of Kentucky, Lexington, KY). The University of Queensland (Herston, QLD, Australia) Molecular Biosciences and Health Sciences Ethics Committees approved all protocols involving animals.

\section{MouseFix Experimental Fracture Model and CSF-1 Treatment}

A standardized femoral fracture model using a flexible plate (MouseFix) was selected in this study for induction of periosteal callus formation via endochondral ossification. The validity and reproducibility of this system as an experimental fracture model has been established. ${ }^{36,37,42,43}$ Mice (aged 11 to 12 weeks) were anesthetized, the left hind limb was shaved, and a longitudinal incision (approximately $12 \mathrm{~mm}$ ) was made along the lateral aspect of the thigh. The lateral fascia and muscles were longitudinally split, and the middle third of the femoral shaft was made accessible to apply the fracture fixation implants. In this area, the surrounding muscles were bluntly dissected from the bone without stripping the periosteum. The flexible plate was attached to the lateral aspect of the femoral shaft using four bicortically placed, head-locking screws. A well-defined, full-thickness osteotomy was generated beneath the flexible plate using a saw guide, resulting in a fracture gap width of $0.22 \mathrm{~mm}$ in the midfemoral shaft. Muscles and skin layers were closed with sutures. No implant failures or change in fracture position was encountered within our series. In some animals, $10 \mu \mathrm{g}$ per mouse bolus dose CSF-1 (PeproTech, Rocky Hill, NJ) or matched vehicle control was administered by injection adjacent to the fracture site (using palpation to determine plate position and subsequently guide injection) every second day, initiating 6 days after fracture. Tissues were harvested from treated mice 11 days after fracture.

\section{Depletion of Macrophages during Fracture Healing Using the Mafia Transgenic Mouse Model}

Mafia transgenic mice, ${ }^{38}$ in which a Fas-based suicide gene expression is driven in mature myeloid cells (predominantly monocytes-macrophages, Csflr promoter), were treated with vehicle (4\% ethanol, $10 \%$ polyethylene glycol, and $2 \%$ Tween-20; Sigma-Aldrich, St. Louis, MO) control or $10 \mathrm{mg} /$ $\mathrm{kg}$ suicide gene activating ligand AP20187 [for some experiments, this was purchased from ARIAD Pharmaceuticals Inc. (Cambridge, MA); in others, it was sourced from Clonetech (Mountain View, CA)] via a direct injection into the fracture osteotomy at the time of surgery $(n=6$ per group) or a retro-orbital injection at 5 days after surgery ( $n=8$ per group). AP20187 ligand was prepared as previously described. ${ }^{28}$ To control for off-target effects or unexpected toxicity of intrafracture AP20187 ligand delivery, C57B1/6 mice $(n=3)$ were treated with AP20187 ligand, as per Mafia mice, at the time of surgery.

\section{Tissue Collection}

All left hind limbs were collected and processed as previously described. ${ }^{14}$ Tissues were embedded to achieve crosssectional sampling of bone in the sagittal plane, with sections ( $5 \mu \mathrm{m}$ thick) cut in a lateral to medial progression. MouseFix model samples in wild-type mice were collected on days 7 (early anabolic), 14 (peak soft callus formation), 21 (peak hard callus formation), and 28 (remodeling). Femora from macrophage depletion experiments were collected on either day 10 to 11 or day 13 after surgery.

\section{Flow Cytometric Analysis}

Bone marrow was harvested from the contralateral, intact femur in macrophage depletion studies, and $1.0 \times 10^{6}$ bone marrow cells were blocked with $0.5 \%$ fetal bovine serum/phosphatebuffered saline before incubation with anti-F4/80-Alexa 647 (AbD Serotec, San Diego, CA) alone or in combination with anti-CD11b-R-phycoerythrin (AbD Serotec) or relevant directly labeled rat IgG2b isotype control antibodies (AbD Serotec). Cells were then washed twice, fixed using $4 \%$ paraformaldehyde (Sigma-Aldrich), and examined by flow cytometry on Gallios or CyAn flow cytometers (Beckman Coulter, Brea, CA) with 50,000 events analyzed. Data were analyzed using Kaluza software version 1.1 (Beckman Coulter) or FlowJo version 8.8.7 (Tree Star Data Analysis Software, Ashland, OR).

\section{Immunohistochemistry}

Immunohistochemistry (IHC) was performed on deparaffinized and rehydrated sections, as previously described, ${ }^{28}$ with specific primary antibodies raised against F4/80 (rat anti-mouse; AbD Serotec), Mac-2 (rat anti-mouse; eBioscience, San Diego, CA), green fluorescent protein (GFP; rabbit anti-jellyfish; Invitrogen, Life Technologies, Grand Island, NY), or relevant isotype control antibody [normal rat IgG2b (AbD Serotec) or normal rabbit IgG fraction (Santa Cruz Biotechnology, Dallas, TX)]. All sections were counterstained using Mayer's hematoxylin (Sigma-Aldrich) and mounted using permanent mounting media (Thermo Fisher Scientific, Waltham, MA). Antigen retrieval was required for anti-F4/80 ( $0.37 \%$ trypsin for 10 minutes) and anti-GFP $(0.05 \mathrm{mg} / \mathrm{mL}$ proteinase $\mathrm{K}$ for 5 minutes $)$. In some cases, dual staining for tartrate-resistant acid phosphatase (TRAP) activity and F4/80 expression was performed as previously described. ${ }^{14}$ Tissue staining was viewed using a Nikon eclipse 80i microscope with a Nikon D5-Ri1 camera and NIS-elements imaging software version 3.1 (Nikon, Tokyo, Japan). 


\section{Histological Staining}

For toluidine blue (Sigma Aldrich) staining, which identifies proteoglycan-rich tissues, such as cartilaginous matrix, dewaxed slides were stained in a $0.1 \%$ toluidine blue solution for 2 minutes and washed in deionized water for 5 minutes. Sections were dehydrated in $100 \%$ ethanol for 10 seconds, followed by two 5-minute 100\% ethanol washes and two 5-minute xylene washes, and then mounted using Cytoseal 60 permanent mounting media (Thermo Fisher Scientific). To identify osteoclasts, dewaxed slides were stained for TRAP, as previously described. ${ }^{44}$ Hematoxylin (Mayer's; Sigma-Aldrich) and eosin (Eosin-Y; Sigma-Aldrich) or Safronin O (SigmaAldrich) staining was performed on dewaxed paraffin sections (5 $\mu \mathrm{m}$ thick) using standard histological protocols.

\section{Quantification of Fracture Soft Callus and Enumeration of Fracture-Associated Macrophages}

Images of hematoxylin and eosin-stained fractures were taken at $\times 10$ magnification using a Nikon eclipse $80 \mathrm{i} \mathrm{mi-}$ croscope. The region quantified was the periosteal fracture zone (Supplemental Figure S1A), which is the periosteal region between the inner proximal and distal screw positions on the non-plated cortex of the femur. The area of soft callus (identified on the basis of histological features of dense matrix containing proliferative and hypertrophic chondrocytes embedded within lacunae) was measured. Soft callus was demarked using the freehand tool in ImageJ software version $1.41 \mathrm{o}$ (NIH, Bethesda, MD), and area values were obtained and converted to $\mathrm{mm}^{2}$ via calibration to a scale bar embedded within each image. For each specimen, the soft callus area was measured at three sectional depths that were at least $50-\mu \mathrm{m}$ apart, and the average value was determined and used for statistical comparisons between groups.

Macrophage and osteoclast quantification was performed in early time course samples that contained granulation tissue with no or minimal evidence of early chondrification centers $(n=6)$. Analysis was restricted to the mesenchymal granulation strata because early anabolic activity initiates within this region. Inflammatory granulation tissue strata that contained many $\mathrm{F} 4 / 80^{+}$macrophages and other infiltrating immune cells were excluded. The numbers of F4/ $80^{+}$and Mac- $2^{+}$macrophage-like cells and TRAP-positive osteoclasts and their precursors were enumerated by manual counting in $\times 20$ magnification images representing approximately half of the periosteal fracture zone (Supplemental Figure S1A), extending from the fracture gap toward the inner proximal or distal screw. In all samples, the full width of the granulation tissue perpendicular to the periosteal surface was within the field of view. Total area of mesenchymal granulation tissue was determined using ImageJ software that was calibrated via a scale bar embedded within each image. The average area of granulation tissue analyzed/sample was $0.15 \mathrm{~mm}^{2}$. Number of positive cells is represented as per $0.1-\mathrm{mm}^{2}$ area of mesenchymal granulation tissue.

$\mathrm{F} 4 / 80^{+}$macrophages were also enumerated in the total periosteal callus (soft and hard callus plus mesenchymal granulation tissue) of delayed AP20187 ligand or vehicletreated Mafia mice. Positively stained cells were manually counted by a blinded assessor (A.R.P.) in composite $10 \times$ images (tiled using photomerge function in Adobe Photoshop; Adobe Systems, San Jose, CA) that included the full primary callus region. Total callus area was measured using ImageJ software, similar to above, and the number of $\mathrm{F} 4 / 80^{+}$ cells $/ \mathrm{mm}^{2}$ of callus was determined.

\section{Statistical Analysis}

Statistically significant differences were determined using unpaired $t$-tests with one- or two-tailed distributions or Mann-Whitney when appropriately indicated by data distribution using PRISM 5 (GraphPad Software, La Jolla, CA). Pearson's correlation, calculated using PRISM 5, was used to determine relationships of two data outputs. $P<0.05$ was deemed statistically significant. In all cases, data are represented as means \pm SEM.

\section{Results}

\section{Fracture-Associated Granulation Tissues Contain Inflammatory Macrophages}

The precise location and phenotype of fracture-associated macrophages have not been clearly established. Nor has it been examined whether this phenotype and distribution shifts throughout the multiple stages of repair. We used the MouseFix flexible plate system to produce a standardized and well-characterized experimental fracture model ${ }^{36,37,42,43}$ in which a femoral diaphyseal osteotomy is stabilized with a flexible plate using locking screws (Supplemental Figure S1A). This model heals by periosteal callus formation via endochondral ossification ${ }^{43}$ (Supplemental Figure S1, B-I). It was chosen because of the high degree of reproducibility in histological features, healing kinetics, and healing magnitude, allowing for fine mapping of endochondral repair events. We focused on periosteal endochondral repair mechanisms associated with the fracture callus and, therefore, restricted analyses to the periosteal injury zone (Supplemental Figure S1A). IHC staining in immediate serial sections for expression of the pan-macrophage marker F4/80, in combination with Mac-2 (alias galectin- $3^{45}$ ), was used to identify resident $\left(\mathrm{F} 4 / 80^{+} \mathrm{Mac}^{\text {neg }}{ }^{\text {ne }}\right)$ versus inflammatory $\left(\mathrm{F} 4 / 80^{+}\right.$Mac$2^{+}$) macrophages. ${ }^{46,47}$ Seven days after surgery, granulation tissue was well established within the periosteal fracture zone and bridged the osteotomy site (Figure 1A and Supplemental Figure S1F). On the basis of morphological features, including density of immune cells, the granulation tissue was stratified into two layers: a mesenchymal granulation layer that was adjacent to the periosteal bone surface and contained a 
predominance of mesenchymal/fibroblast-like cells and an inflammatory layer, which was the superior layer containing a high density of immune cells, including polymorphonuclear cells (Figure 1).

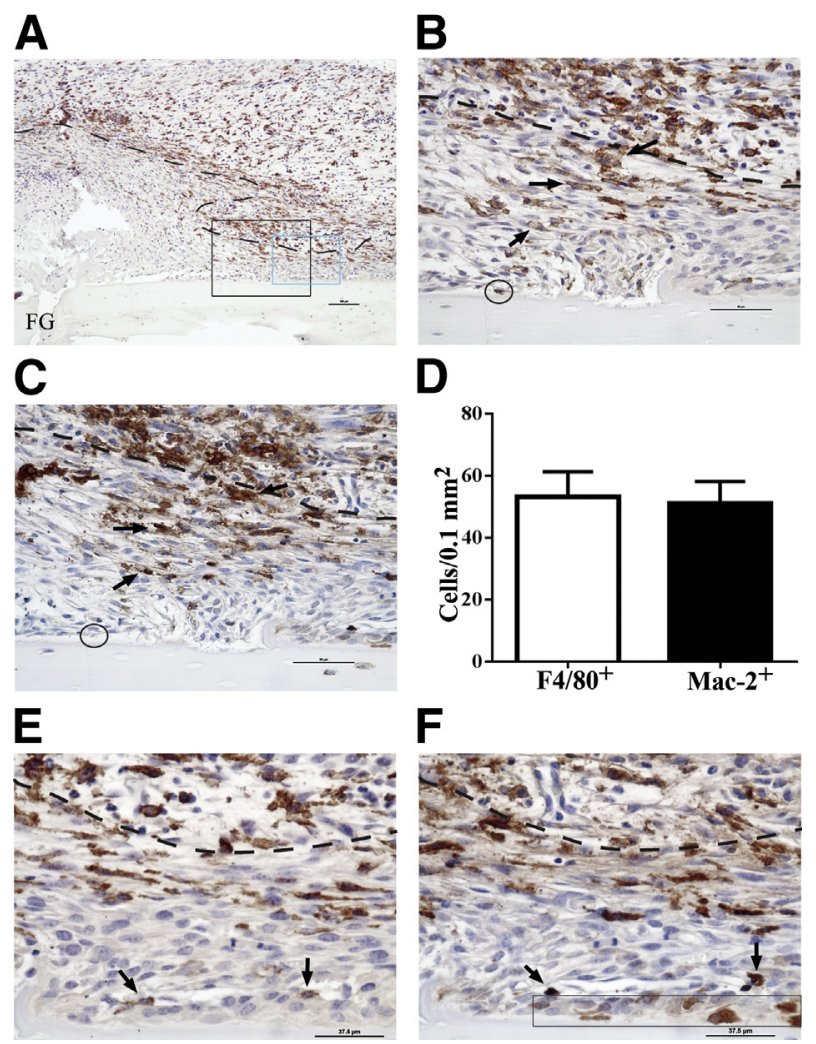

Figure 1 Inflammatory macrophages predominate in fracture granulation tissue and associate with chondrification centers. Representative images of periosteal fracture zone (Supplemental Figure S1A) 7 days after osteotomy and MouseFix plate fixation surgery in 12-week-old C57Bl/6 mice ( $n=6$ with assessment at multiple sectional depths per sample). All sections were counterstained with hematoxylin. A: Image represents approximately half of the periosteal fracture zone (proximal) with the osteotomy-generated fracture gap $(\mathrm{FG})$ at the bottom left of the image. Granulation tissue predominates at this time point. Tissue section was stained by IHC for the F $4 / 80$ pan-macrophage antigen (brown). The black box demarks the region shown in $\mathbf{B}$ and $\mathbf{C}$. The blue box demarks the region shown in $\mathbf{E}$ and $\mathbf{F}$. B: $\mathrm{IHC}$ for $\mathrm{F} 4 / 80$ expression within fracture granulation tissue. The dashed line demarks the interface between the mesenchymal (closest to bone, below dashed line) and inflammatory (above dashed line) stratum. $\mathrm{F} 4 / 80^{+}$cells with stellate morphological characteristics are evident in both the inflammatory and mesenchymal (arrows) strata. The circle demarks a single osteomac within this field. C: IHC for Mac-2 expression (brown) in a serial section to that shown in B. Arrows point to the same cells indicated in $\mathbf{B}$ and highlight the high degree of overlap in F4/80 (B) and Mac-2 (C) staining patterns. The circle indicates the Mac- $2^{\text {neg }}$ osteomac identified in B. D: Quantification of the number of $\mathrm{F} 4 / 80^{+}$and macrophage-like Mac- $2^{+}$cells within the mesenchymal stratum of the granulation tissue. An average area of $0.15 \mathrm{~mm}^{2}$ was assessed in six independent samples, and the number of positive cells was not statistically different. E: $\mathrm{F} 4 / 80^{+}$macrophages (brown, arrows) adjacent to a periosteal chondrification center. F: Mac-2 staining in a serial section to $\mathbf{E}$ confirms induction of Mac-2 expression in condensing chondrocyte-like cells within the periosteal chondrification center (blue boxed area). The same $\mathrm{F} 4 / 80^{+}$macrophages noted in $\mathbf{E}$ can be traced and express Mac-2 (arrows). Dashed line in B, C, E, and $\mathbf{F}$ demarks the mesenchymal (lower)- inflammation (upper) strata junction within the granulation tissue. Original magnifications: $\times 10(\mathbf{A}), \times 40(\mathbf{B}$ and $\mathbf{C}) ; \times 60(\mathbf{E}$ and $\mathbf{F})$. Scale bars: 100 $\mu \mathrm{m}(\mathbf{A}) ; 50 \mu \mathrm{m}$ (B and $\mathbf{C}) ; 37.5 \mu \mathrm{m}$ (E and $\mathbf{F})$.
Both overall anti-F4/80 and anti-Mac-2 antibody staining patterns and tracing of individual cells within the serial sections confirmed a predominance of $\mathrm{F} 4 / 80$ and Mac-2 coexpression in the fracture callus. The inflammatory layer contained abundant $\mathrm{F} 4 / 80^{+} \mathrm{Mac}-2^{+}$ inflammatory macrophages (Figure 1, A-C) and lymphocyte-like and polymorphonuclear granulocyte-like cells (Figure 1, B and C). The mesenchymal layer had minimal infiltration of cells with lymphocyte or granulocyte morphological features, but contained scattered $\mathrm{F} 4 / 80^{+}$Mac-2 $2^{\text {high }}$ inflammatory macrophages (Figure 1, B and C). Quantification of the number of $\mathrm{F} 4 / 80^{+}$cells and Mac- $2^{+}$macrophage-like cells within the mesenchymal granulation tissue demonstrated almost identical density of these populations (Figure 1D), confirming that most macrophages within the tissue display an inflammatory phenotype.

At day 7 after surgery, evidence of early anabolic repair (soft callus formation) could be detected within the periosteal fracture zone. This occurred bilaterally, a short distance from the osteotomy (approximately $750 \mu \mathrm{m}$ ) as proteoglycan-rich chondrification centers adjacent to the periosteal surface within the mesenchymal granulation tissue (Figure 1, E and F), evidenced by toluidine blue staining (Supplemental Figure S1F) (data not shown). As described previously, ${ }^{48,49}$ chondroblasts were identified as $\mathrm{F} 4 / 80^{\text {neg- }}$ Mac- $2^{+}$condensed mesenchymal cells (Figure 1F). F4/ $80^{+} \mathrm{Mac}^{+}{ }^{+}$(Figure 1, E and F) inflammatory macrophages were observed adjacent to chondrification centers and associated vascular structures. Few, if any, TRAP-positive osteoclast-like cells (data not shown) and only occasional cells meeting osteomac criteria $\left(\mathrm{F} 4 / 80^{+} \mathrm{Mac}-2^{\text {neg }}\right.$ cells within a three-cell distance of bone) (Figure 1, B and C) were observed within the granulation tissue at this time point. A more mature chondrification center is shown in Supplemental Figure S2, demonstrating an expanding proteoglycan-rich matrix (Supplemental Figure S2A) encasing Mac- $2^{+}$chondrocytes (Supplemental Figure S2B). Similar to observations in a previous report, ${ }^{17} \mathrm{~F} 4 / 80^{+}$ macrophages were excluded from the expanding cartilage core, but scattered $\mathrm{F} 4 / 80^{+} \mathrm{Mac}-2^{+}$inflammatory macrophages were present in the immediately adjacent mesenchymal granulation tissue (Supplemental Figure S2, B and C). Overall, the mesenchymal granulation tissue contained inflammatory macrophages that associated with early anabolic events.

\section{Macrophage Depletion in Mafia Transgenic Mice Prevents Fracture-Associated Granulation Tissue Formation in a Femoral Fracture Model}

The absolute requirement of macrophages in endochondral callus formation has not been definitively tested. Therefore, we investigated the consequence of macrophage depletion, using the inducible Mafia transgenic mouse model in conjunction with our chosen experimental femoral fracture 

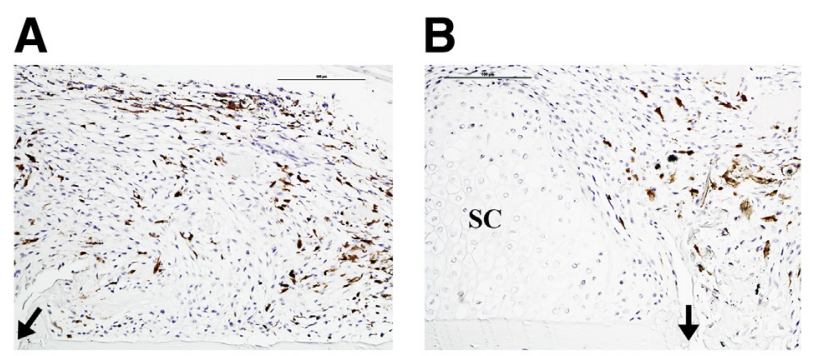

C

D
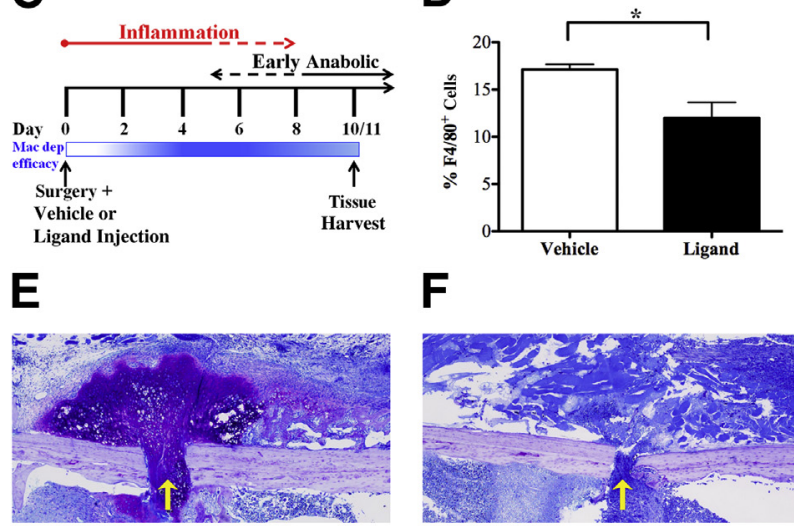

G

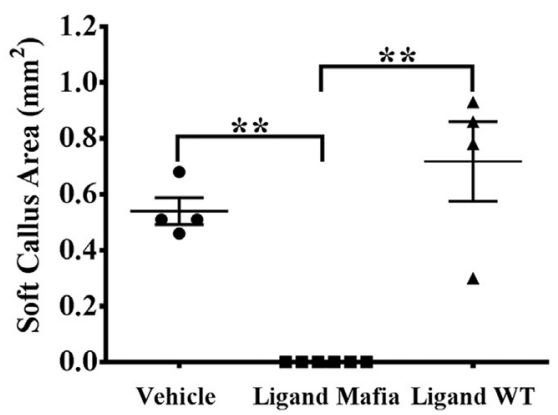

Figure 2 Fracture repair fails in a standardized femur fracture model when macrophage depletion is initiated at the time of fracture surgery. IHC staining for transgenic GFP expression (brown chromogen) in mesenchymal granulation tissue (A) and early soft callus (SC) formation (B) in vehicle-treated Mafia transgenic mice. Black arrows indicate the fracture gap in cortical bone, which is located at the bottom of the field of view in both images. C: Schematic representing the experimental regimen. Schematic components from top to bottom are as follows: fracture phase timing, timeline starting at day 0 , heat map representation of anticipated macrophage depletion efficacy (Mac dep efficacy) with deep blue indicating greatest depletion efficiency, and timing of the interventions (surgery, AP20187 ligand delivery, and harvest). Data are representative of the following group sizes: ligand, $n=5$ in $\mathbf{B}$, and $n=6$ in $\mathbf{C}-\mathbf{E}$ or vehicle $(n=4$ in $\mathbf{B}-\mathbf{E})$. D: Flow cytometric analysis of $\mathrm{F} 4 / 80^{+}$macrophage frequency in contralateral limb bone marrow at the time of tissue harvest. Significance was determined using a one-tailed unpaired $t$-test. Representative images of the periosteal injury zone stained with toluidine blue from vehicle (E) or AP20187 ligand (F) treated mice. Yellow arrows indicate cortical fracture gap generated by osteotomy. G: Quantification of soft callus area $\left(\mathrm{mm}^{2}\right)$ in vehicle-treated Mafia (circles), AP20187 ligand-treated Mafia (squares), and AP20187 ligand-treated C57Bl/6 wild-type (WT; triangles) mice. As required because of data distribution, significance was determined using a MannWhitney analysis. There is no significant difference in soft callus area between AP20187 ligand-treated Mafia and C57Bl/6 mice. ${ }^{*} P<0.05$, ${ }^{*} P<0.005$. Original magnifications: $\times 20(\mathbf{A}$ and $\mathbf{B}) ; \times 4(\mathbf{E}$ and $\mathbf{F})$. Scale bars: $100 \mu \mathrm{m}$ (A and $\mathbf{B})$. model. IHC staining for GFP expression, which is part of the Mafia suicide transgene construct, demonstrated similar distribution of GFP expression in early mesenchymal granulation tissue (Figure 2A) and at early soft callus foci (Figure 2B), as seen for anti-F4/80 ${ }^{+}$staining (Figure 1 and Supplemental Figure S2). There was no expression of GFP and, consequently, the suicide transgene in granulation tissue fibroblasts/mesenchymal cells or soft callus chondrocytes (Figure 2, A and B). Macrophage depletion was initiated at the time of surgery via direct injection of AP20187 ligand into the osteotomy site (Figure 2C). On the basis of extensive experimental testing within the in-house Mafia colony, we anticipated this injection regimen would achieve profound bone marrow and fracture-associated macrophage depletion approximately 2 days after injection (Figure 2C) (data not shown). Bone marrow from the contralateral limb was used to evaluate macrophage number and subsequent depletion efficacy via flow cytometry. Similar to our previous study, ${ }^{14}$ the single intraosteotomy injection of AP20187 ligand at the time of fracture surgery induced systemic depletion of $\mathrm{F} 4 / 80^{+}$bone marrow macrophages, which was sustained at the experimental end point (Figure 2D). Because macrophage numbers recover after AP20187 ligand treatment, the magnitude of depletion at the end point of this experiment, within a tissue that was unlikely to have received an optimal dose of AP20187 ligand, was expectedly conservative but significant $(P<0.05)$.

At day 10 to 11 after surgery, the fractured limb was harvested and radiographically assessed before processing for histological analysis. As expected at this early anabolism time point (ie, predominance of soft callus formation), X-ray imaging indicated minimal formation of mineralized callus in any of the samples (data not shown). Histological assessment of toluidine blue staining to differentiate proteoglycan-rich cartilaginous matrix demonstrated that vehicle-treated mice developed robust periosteal calluses composed of proteoglycan-rich cartilage that fully bridged the fracture (Figure 2E). At this time point, cartilaginous callus predominated, but woven bone-like matrix was present at the proximal edge of the callus (Figure 2E), indicating disequilibrium in callus maturation rate. Macrophage depletion initiating at the time of surgery resulted in catastrophic failure in fracture repair (Figure 2F). Cartilage and woven bone formations were absent within the periosteal fracture zone (Figure 2F). The periosteal bone surface was devoid of any tissue resembling either granulation tissue or periosteum, with only residual hematoma evident in the medullary cavity and the osteotomy gap and partially extruding into the periosteal fracture zone (Figure 2F). Muscle fibers were observed in the periosteal fracture zone (Figure 2F), presumably occupying this region as a consequence of granulation tissue absence. Quantification of total soft callus area in both vehicle and AP20187 ligandtreated Mafia mice, and AP20187 ligand-treated C57B1/6 mice, confirmed that macrophage depletion initiating at the time of surgery abolished soft callus formation $(P=0.003)$ (Figure 2G). 
Macrophage Phenotype and Distribution throughout Progression from Early to Late Anabolic Phases during Endochondral Callus Formation in a Femoral Fracture Model

We next assessed macrophage distribution at the chondroosseous junction during the conversion of soft-to-hard callus and within the maturing hard callus. Vascular invasion into cartilage is an important step in soft-to-hard callus conversion. ${ }^{50}$ Invading vasculature was identifiable within the callus cartilage in femoral fracture samples containing mature soft callus (Figure 3A) with minimal or only partial hard
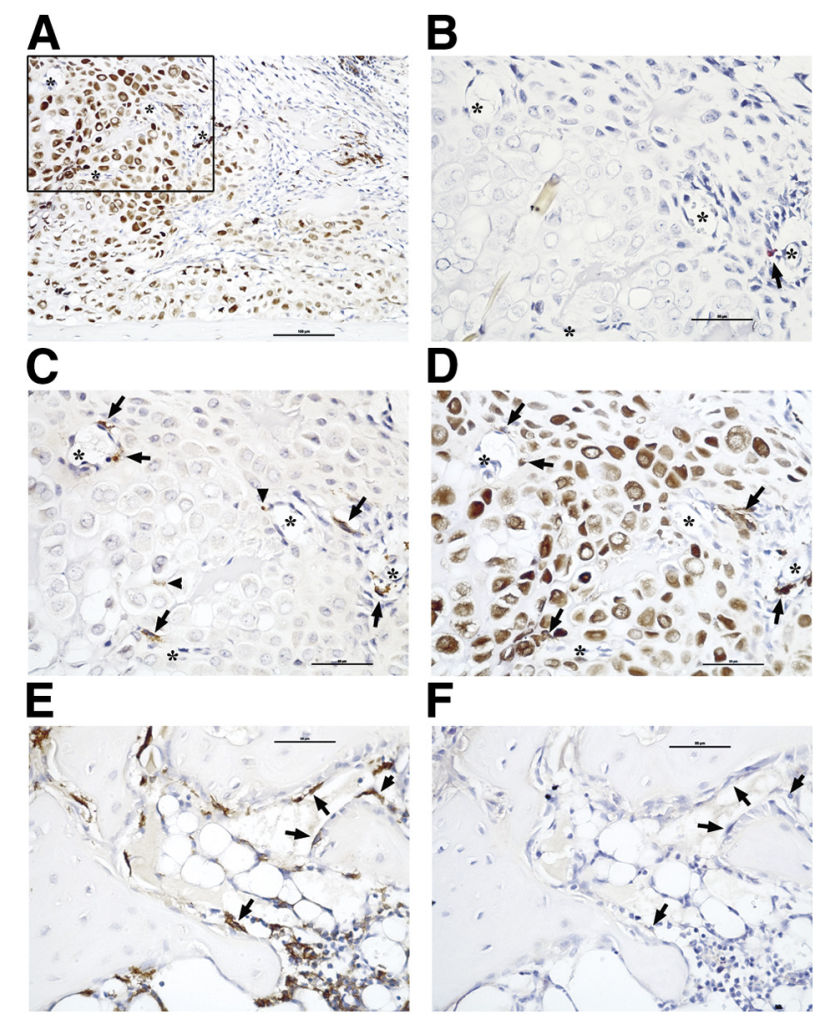

Figure 3 Different macrophage subpopulations localize to regions of soft-to-hard callus transition and hard callus maturation. IHC staining (brown) for F4/80 (C and E) and Mac-2 (A, D, and F) expression in early $(\mathbf{A}-\mathbf{D})$ and late (E and $\mathbf{F})$ anabolic phases of endochondral callus formation. All tissues were counterstained with hematoxylin. A-D: A sample harvested at 11 days after surgery. $\mathbf{E}$ and $\mathbf{F}$ : A sample harvested 21 days after surgery. The boxed area in $\mathbf{A}$ is represented at higher magnification in B-D. Asterisks in A-D track the same blood vessels in all images. A: Mac2 staining demonstrates advanced maturation of soft callus containing $\mathrm{Mac}^{+} 2^{+}$chondrocytes within lacunae. B: Histological staining for TRAP activity demonstrates few TRAP ${ }^{+}$cells were present within the callus at this repair stage. Staining in serial sections for F4/80 (C) and Mac-2 (D) expression. At high magnification, vascular canals (asterisks in $\mathbf{C}$ and $\mathbf{D}$ ) containing $\mathrm{F} 4 / 80^{\text {neg }} \mathrm{Mac}-2^{\text {neg }}$ endothelial-like cells are present within the soft callus cartilage. $\mathrm{F} 4 / 80^{+} \mathrm{Mac}-2^{+}$inflammatory macrophages (arrows) are associated with the vascular canals. Staining in serial sections for F4/80 (E) and Mac-2 (F) expression within hard callus (Supplemental Figure S3). Comparison of staining patterns between $\mathbf{E}$ and $\mathbf{F}$ illustrates the predominance of $\mathrm{F} 4 / 80^{+} \mathrm{Mac}-2^{\text {neg }}$ resident macrophages, including osteomacs (arrows) within the maturing hard callus. Few Mac-2-expressing macrophages or chondrocytes are located within the hard callus. Original magnifications: $\times 20(\mathbf{A}) ; \times 40(\mathbf{B}-\mathbf{F})$. Scale bars: $100 \mu \mathrm{m}(\mathbf{A}) ; 50 \mu \mathrm{m}(\mathbf{B}-\mathbf{F})$. callus conversion (post-surgery days 10 to 14 , seven samples assessed). Vascular identification was based on morphological characteristics (including presence of a lumen and/or red cell ghost) and absence of F4/80, Mac-2, or TRAP expression on endothelial-like cells (Figure 3, A-D). Only rare TRAP ${ }^{+}$ mononuclear or osteoclast-like cells were observed in the callus at this stage of maturation (Figure 3B) or at the immediate chondro-osseous junction in more mature calluses (data not shown). In virtually all cases, $\mathrm{F} 4 / 80^{+}$macrophage(s) or their $\mathrm{F} 4 / 80^{+}$cellular processes (Figure 3C) were associated with the vascular invasions within the cartilaginous callus. Staining in serial sections clearly demonstrated that these macrophages, which were traceable despite being in a field of $\mathrm{Mac}_{-} 2^{+}$chondrocytes, also expressed Mac-2 (Figure 3D), supporting their status as inflammatory macrophages.

Macrophage subtype and distribution were more complex within callus undergoing soft-to-hard callus transition. First, macrophages were abundant within the periosteal-like tissue encapsulating the maturing callus (Supplemental Figure S3, A and $\mathrm{C}$ ). Comparison to Mac-2 staining (Supplemental Figure S3B) indicated both $\mathrm{F} 4 / 80^{+} \mathrm{Mac}-2^{\text {neg }}$ resident and $\mathrm{F} 4 /$ $80^{+} \mathrm{Mac}^{-} 2^{+}$inflammatory macrophages were distributed within this encapsulating tissue. Second, both inflammatory and resident macrophages were also present at the chondroosseous junction at sites of established soft-to-hard callus transition (Supplemental Figure S3A) (data not shown). Macrophages were more common than osteoclasts at the chondroosseous interface (Supplemental Figure S3, A and D) (data not shown). Finally, within the maturing hard callus (days 14 to 21 after surgery, eight samples assessed), both $\mathrm{F} 4 / 80^{+}$macrophages and TRAP $^{+}$osteoclasts were common (Supplemental Figure S3, A, C, and D). Within the new woven bone matrix, $\mathrm{F} 4 / 80^{+}$macrophages were present within the pseudohematopoietic tissue associated with the woven bone (Figure 3E). Elongated $\mathrm{F} 4 / 80^{+}$macrophages were also associated with cuboidal osteoblast-like cells on woven bone surfaces (Figure 3E). Staining in a serial section demonstrated minimal Mac-2 expression in the woven bone callus (Figure $3 \mathrm{~F}$ and Supplemental Figure S3B), indicating that the macrophages within the actively forming bone are predominantly resident macrophages, including osteomacs (Figure 3, E and F).

\section{Macrophage Depletion Coinciding with Initiation of Early Anabolism in the MouseFix Internally Fixed Femoral Fracture Model Significantly Impairs Soft Callus Formation}

We aimed to more clearly delineate between the now confirmed vital contributions of macrophages to early inflammatory processes and granulation tissue formation (Figures 1 and 2) versus their less established role in anabolic events during endochondral callus formation. AP20187 ligand administration in Mafia transgenic mice that had diaphyseal femoral fractures was delayed until 5 days after 
surgery and delivered by a single systemic injection (Figure 4A). Day 5 was chosen for initiation of ligand delivery for the following reasons: fully established granulation tissue is present at day 5 after surgery ${ }^{43}$ (data not shown); and previous depletion kinetics indicated that peak macrophage depletion in bone marrow is achieved 48 hours after a single systemic injection of AP20187 ligand (data not shown). Consequently, our approach will allow the inflammatory phase to complete with minimal impediment but significantly affect macrophages at the transition to and throughout the early anabolic phase.
Flow cytometric analysis of bone marrow from the contralateral femur confirmed significant and robust reduction in F4/ $80^{+}$mature bone marrow macrophages $(P<0.0001)$ (Figure 4B) at the experimental end point. Vehicle-treated mice had a robust periosteal callus composed of proteoglycan-rich cartilage bridging the fracture site with woven bone often present at either or both the distal and proximal edges of the callus (Figure 4C). In macrophagedepleted mice, variation in the magnitude of fracture repair was observed. Some animals exhibited a small, but morphologically normal, callus (Figure 4D), whereas others had
A

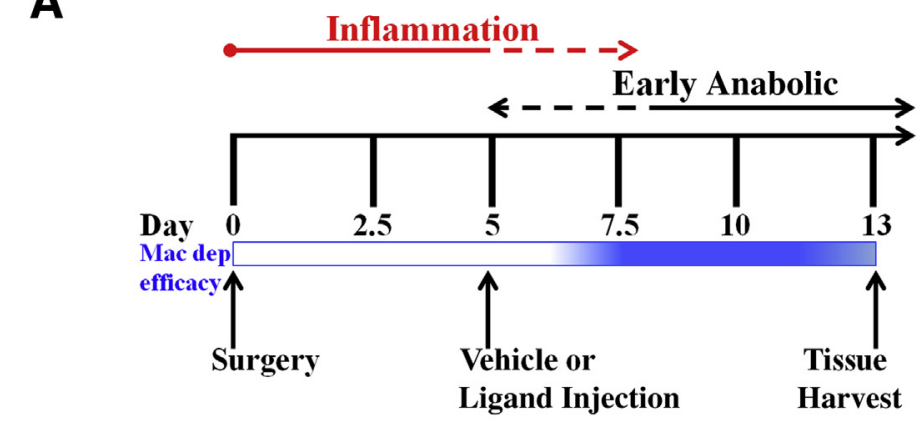

Ligand Injection

B

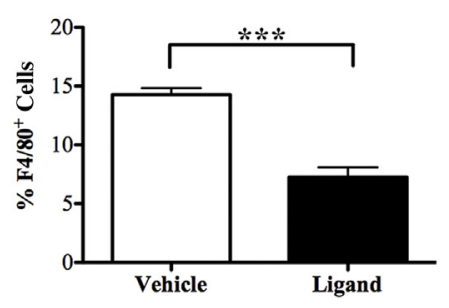

C

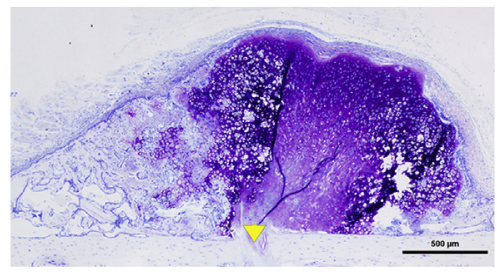

$\mathbf{F}$

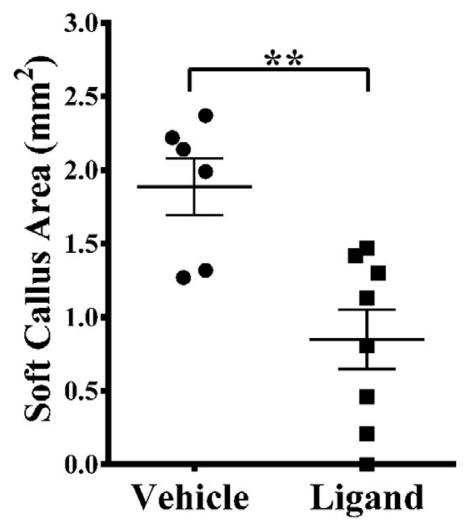

D

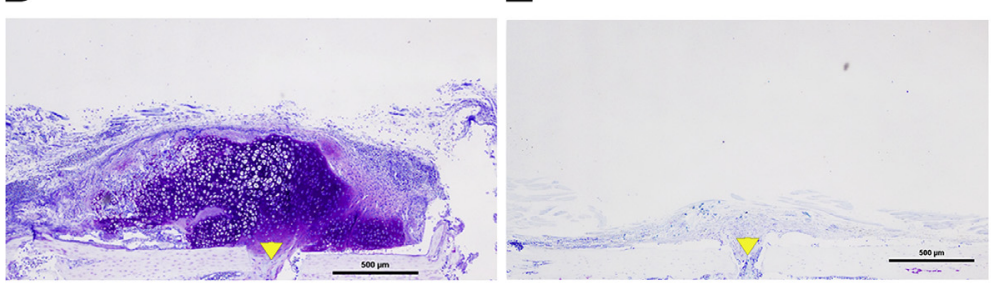

G

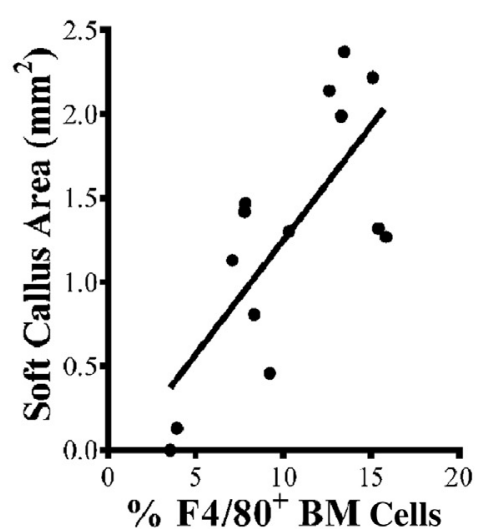

H

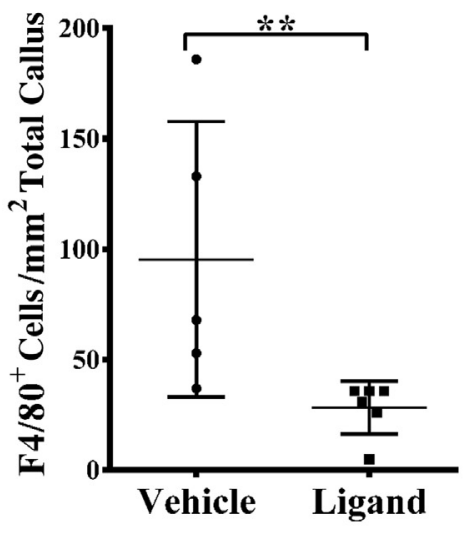

Figure 4 Delayed depletion of macrophages compromises periosteal endochondral fracture repair. Data represent MouseFix plated femoral fracture model treated with either AP20187 ligand $(n=8)$ or vehicle $(n=6)$. A: Schematic outlining the experimental regimen. Schematic components from top to bottom are as follows: fracture phase, timeline starting at day 0 , heat map style representation of anticipated macrophage depletion efficacy (Mac dep efficacy) with deep blue indicating greatest depletion efficacy, and timing of interventions (surgery, AP20187 ligand delivery, and harvest). B: Bar graph represents flow cytometric analysis of $\mathrm{F}_{4} / 80^{+}$macrophage frequency in contralateral limb bone marrow. Significance was determined using a one-tailed unpaired $t$-test. Representative images of the periosteal injury zone stained with toluidine blue from vehicle (C) or AP20187 ligand (D and E) treated mice. Arrowheads indicate cortical fracture gap generated via osteotomy, and the proximal aspect is always on the left side of the image. F: Quantification of soft callus area $\left(\mathrm{mm}^{2}\right)$ in vehicle (circles) and AP20187 ligand (squares) treated mice. Significance was determined using a one-tailed unpaired $t$-test. G: Spearman correlation

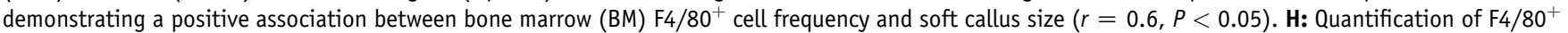
macrophages per area $\left(\mathrm{mm}^{2}\right)$ of total periosteal callus in vehicle (circles) and AP20187 ligand (squares) treated mice (Mann-Whitney analysis). ${ }^{* *} P<0.005,{ }^{* * *} P<0.0001$. Original magnification, $\times 4(\mathrm{C}-\mathrm{E})$. 
minimal or no soft callus formation (Figure 4E). Quantification of soft callus area in both vehicle and AP20187 ligand-treated mice confirmed that delayed macrophage depletion significantly reduced soft callus size $(P<0.005)$ (Figure $4 \mathrm{~F})$. Callus size positively correlated with the percentage of $\mathrm{F} 4 / 80^{+}$bone marrow macrophages in the contralateral limb $(r=0.6$, $P<0.05$ ) (Figure $4 \mathrm{G}$ ). IHC analysis indicated that when callus formed in AP20187-treated mice, $\mathrm{F} 4 / 80^{+}$macrophages were present and exhibited normal distribution for the fracture stage, but at significantly lower frequency than observed in vehicletreated mice $(P<0.005)$ (Figure $4 \mathrm{H})$. Overall, confining the depletion of macrophages until after the establishment of normal inflammatory events had significant impact on progression and/or magnitude of anabolism during endochondral callus formation.

Treatment with the Myeloid-Trophic Cytokine CSF-1 during the Early Anabolic Phase of Endochondral Callus-Mediated Bone Repair Enhances Soft Callus Formation

CSF-1 is a myeloid proliferation and differentiation factor and, in periphery, promotes migration, proliferation, and survival of mature myeloid cells, particularly macrophages and osteoclasts. ${ }^{39,51}$ We used local delivery of CSF-1, initiating 6 days after fracture, to specifically assess the outcome of enhancing macrophages during the early anabolic phase on fracture healing. Tissue was harvested day 11 after surgery to allow assessment of both increased magnitude of soft callus formed and/or rate of anabolic progression. As expected, vehicle-treated mice had an immature, relatively small callus that had clear bilateral chondrification foci, including an identifiable soft callus (Figure 5A). CSF-1-treated mice had a markedly increased amount of soft callus (Figure 5B), but there was no histological indication of accelerated healing (premature bridging or conversion to hard callus). Double staining for TRAP activity and F4/80 expression supported enhanced macrophage frequency within the callus granulation tissue of CSF-1 - treated mice, with no obvious impact on TRAP ${ }^{+}$ cell number or distribution (Figure 5, C and D). Quantification of soft callus area confirmed a significant twofold increase in soft callus formation in CSF-1-treated mice compared with vehicle controls (Figure 5E). These data indicate that pro-macrophage effects of CSF-1 therapy resulted in enhanced soft callus formation during endochondral fracture healing.

\section{Discussion}

This study expands on previous observations regarding macrophage involvement in fracture repair, which includes novel observations of macrophage distribution in later anabolic stages and broad subcategorization of macrophage phenotypes. Similar to previous reports, ${ }^{14-17,52}$ in the

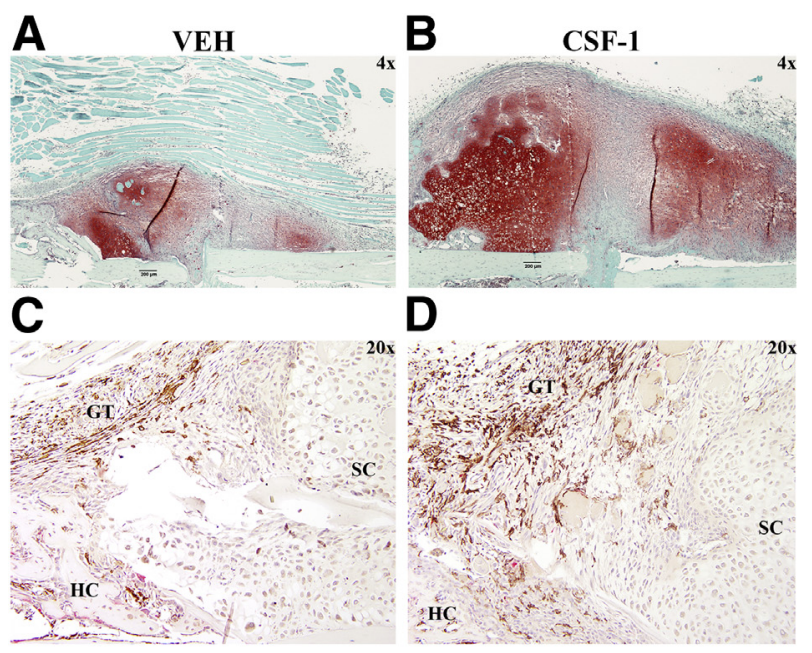

E

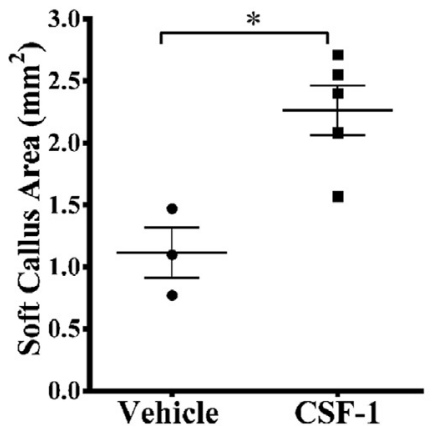

Figure 5 Delayed CSF-1 treatment restricted to the early anabolic phase enhances soft callus formation. Data represent a MouseFix plated femoral fracture model treated with either CSF-1 $(n=5)$ or vehicle $(n=3)$. Treatment was initiated 6 days after fracture (transition from inflammatory to early anabolic phase) and was given via local injection every second day until tissue harvest at day 11. A and B: Safronin 0 histological staining (cartilage stains orange-red, green counterstain) shows representative soft callus formation in vehicle (VEH; A) or CSF-1 (B) treated mice. C and D: Double staining for TRAP (magenta) activity and F4/80 (brown) expression demonstrates osteoclast and macrophage distribution and frequency, respectively, at the callus periphery in representative vehicle (C) and CSF-1 (D) treated mice. Field of view was selected because it contains granulation tissue (GT), soft callus (SC), and hard callus (HC). Sections were counterstained with hematoxylin. E: Quantification of soft callus area $\left(\mathrm{mm}^{2}\right)$ in vehicle (circles) and CSF-1 (squares) treated mice. Significance was determined using a one-tailed unpaired Mann-Whitney test. ${ }^{*} P<0.05$. Original magnifications: $\times 4$ (A and B); $\times 20$ (C and D). Scale bars: $200 \mu \mathrm{m}$ (A and B).

experimental femoral fracture model used here, macrophages were a prominent cell population during the inflammatory phase of fracture healing. We extended previous observations by demonstrating that most of the granulation tissue macrophages display an inflammatory phenotype $\left(\mathrm{F} 4 / 80^{+} \mathrm{Mac}-2^{+}\right)$. We noted that the granulation tissue was stratified. An inflammatory stratum contained a high density of inflammatory macrophages and is likely responsible for prolonged damage control, including removal of tissue/cell debris and antimicrobial functions. Less expected was the presence of inflammatory macrophages in the mesenchymal stratum of the granulation tissue, including some that were associated with 
developing chondrification centers. Inflammatory macrophages were also present within the granulation tissue immediately adjacent to the expanding soft callus front, confirming a previous report in an alternative fracture model. ${ }^{17}$ This cellular distribution provides circumstantial evidence for direct participation of inflammatory macrophages in the initiation of endochondral callus formation. Notably, markedly increased soft callus formation in fractures treated with CSF-1 during the early anabolic phase provided strong evidence that macrophages provide pro-anabolic support to soft callus formation during fracture healing.

Inflammatory macrophages also localized with vascular invasions in the mature soft callus. The histological features of the vascular invasions were not reminiscent of vascular canals that have been previously reported within the secondary ossification centers during developmental endochondral ossification. ${ }^{53}$ The vascular invasions noted herein were narrow blood vessel tracks that contained only vasculature and associated inflammatory macrophages, similar to blood vessel channels through mature bone. ${ }^{28}$ The developmental vascular canals were broad invaginations with osteoclasts/chondroclasts at the apical tip, presumably excavating the canal path via matrix degradation, followed by a mixture of mesenchymal cells, osteoclasts/chondroclasts, lysosomal cells, macrophages, and endothelial cells. ${ }^{53}$ The presence of macrophages within the less complex vascular invasions into mature soft callus provides a potential cellular mechanism to explain why soft callus continues to become vascularized when osteoclast activity is prevented. ${ }^{54}$ Cartilage canal-like structures were present within established periosteal callus chondro-osseous junctions when softto-hard callus transition was in full progression and contained numerous macrophages, both inflammatory and resident type, as well as osteoclasts. More detailed investigation is required to determine whether there is variation in the positional hierarchy of cells within these canals compared with those described in developmental endochondral ossification. ${ }^{53}$ Taken together, evidence is mounting in support of macrophages playing a role in vital processes ${ }^{54,55}$ required for the progression of endochondral ossification from cartilaginous to bony matrix formation in both developmental and bone repair settings.

In the late anabolic phase of our chosen experimental fracture model, we demonstrated the presence of predominantly resident macrophages, including osteomacs, within the maturing/remodeling hard callus. The osteomacs overlaid cuboidal osteoblast-like cells on woven bone surfaces. This macrophage phenotype and distribution recapitulated our observations in a tibial injury model. At the peak anabolic phase, the tibial injury model had a predominance of resident macrophages/osteomacs within the de novo bone bridging the injury site. This tibial injury model heals via intramembranous ossification and macrophages, but without osteoclast requirement for optimal bone formation. ${ }^{14}$ The overall cellular distribution, including that of macrophages, and the anabolic mechanisms occurring at the late anabolic phase of the current endochondral ossification repair model have high similarity to the intramembranous repair process we have reported in a tibial injury model. These similarities are suggestive of the importance of macrophage contributions to the late anabolic phase of endochondral callus formation. Overall, the detailed mapping of macrophage distribution during fracture repair via endochondral callus formation supports that macrophages make prolonged and diverse contributions throughout the healing response.

Data presented herein make progress by broadly classifying the type of macrophages involved in fracture healing. A changing of the guard from inflammatory to resident tissue macrophage occurred when progressing from early to late anabolic phases. This supports the hypothesis that different macrophages mediate/influence specific events during endochondral callus formation. In-depth profiling of macrophage phenotype during fracture repair will be vital to understand the functional contributions of these cells. ${ }^{31,56,57}$ Recruitment/expansion of resident versus inflammatory macrophages, and polarization of the latter toward either a M1 or M2 phenotype,${ }^{58}$ is likely to affect functional contributions of fracture-associated macrophages. ${ }^{33}$ Emerging data support that macrophage activation bias affects their functional input to bone biology. ${ }^{31-33,59}$ However, they provide no clear consensus with respect to the benefit/detriment of M1 versus M2 polarization. Ongoing investigation is required to determine whether the different macrophage subpopulations have divergent influences on specific events required for anabolic repair.

The current study provides the first direct demonstration that macrophages are required for initiation of endochondral callus formation. The consequence of macrophage depletion initiating from the time of surgery was absolute, with universal failure in initiation of granulation tissue formation. This confirms the previously implied broad spectrum of contributions made by macrophage to early repair events. ${ }^{9-11}$ Previous studies have provided indirect evidence in this regard through manipulation of macrophage recruitment and/or macrophage-expressed molecules. ${ }^{15,17,19,20}$ Most notably, examination of fracture repair in chemokine receptor type 2-deficient mice showed a significant reduction of F4/ $80^{+}$macrophages in fracture-associated tissues 3 days after injury. ${ }^{15}$ Concomitantly, there was a significant reduction in total callus area 7 days after injury. ${ }^{15}$ Counterintuitively, the areas of fracture-associated new bone and cartilage were similar in wild-type and chemokine receptor type 2-deficient mice, ${ }^{15}$ suggesting that the significant change in total callus area was due to differences in soft tissue volume. The fracture model used was a blunt trauma three-point bending model with no stabilization or fracture reduction. ${ }^{15}$ This model would have a large degree of ongoing soft tissue damage and, therefore, is likely biased toward recruitment of and dependence on inflammatory macrophages.

This study has provided the first direct evidence that macrophages participate in the anabolic mechanism involved in endochondral callus formation during fracture repair 
progression. Impaired soft callus formation was observed in the used femoral fracture model when macrophage depletion was timed to coincide with initiation of anabolic events, while preserving their contribution to the inflammatory phase. The ability of macrophages to support chondrogenesis has not been broadly examined and warrants further investigation. Macrophages and chondrocytes certainly communicate via paracrine mechanisms during inflammation-associated cartilage degradation, ${ }^{60}$ and M1 macrophages derived from osteoarthritic synovium may have anti-chondrogenic activity. ${ }^{61}$ However, older data indicate that macrophages are pro-mitogenic toward chondrocytes. ${ }^{62}$ It is possible that the delayed macrophage depletion strategy did affect ongoing/resolving inflammatory processes that overlap with the anabolic phase of repair. However, the direct involvement of macrophages in fractureassociated chondrogenesis and chondrogenic function is strengthened when coupled with our detailed macrophage distribution pattern.

A limitation of the Mafia model is that, because of reliance on Csfl-r promoter expression, ${ }^{38}$ depletion of offtarget myeloid cells can occur, including osteoclasts, ${ }^{41}$ granulocytes, ${ }^{29,63}$ and mature dendritic cells. ${ }^{64} \mathrm{We}$ were unable to identify any studies implicating dendritic cell biological characteristics in local fracture repair mechanisms and, thus, the potential impact of off-target deletion of this myeloid cell is unknown. With respect to osteoclast involvement in the experimental femoral fracture model used herein, TRAP-positive mononuclear or multinucleated cells were rarely observed within the granulation tissue, implying minimal functional contribution of osteoclasts to early repair events. Also, strategies that specifically target osteoclasts in other fracture models do not phenocopy the observations made herein, ${ }^{65-69}$ indicating osteoclast depletion does not underlie the current observations. However, further experimentation in the chosen fracture model is required to definitively eliminate potential osteoclast contributions. Finally, neutrophil granulocytes are present in similar numbers to macrophages in fracture-associated tissues early after fracture (day 3), with macrophages predominating thereafter. ${ }^{70}$ In the femoral fracture model used herein, neutrophil granulocytes were present in the granulation tissue at both days 5 and 7 after surgery. However, they were predominantly restricted to the inflammatory granulation stratum, rather than the mesenchymal stratum. Although the distribution does not support a role in anabolic events, we cannot rule out that off-target depletion of these cells has contributed to the current observations.

Macrophages are appropriately located within fractureassociated tissues and can express the required molecular repertoire to initiate, influence, and coordinate both inflammatory processes and bone anabolic outcomes. ${ }^{71}$ Overall, this study clarifies the importance, range, and duration of macrophage contributions to endochondral bone repair. It also highlights that multiple macrophage subtypes are involved in the repair mechanisms. We propose that macrophages represent a viable target for therapeutically enhancing fracture healing, with proof-of-concept provided by CSF-1 treatment, with a potentially broad therapeutic window of opportunity. Finally, as suggested recently using a muscular dystrophy model, ${ }^{72}$ compromised/altered macrophage biological characteristics should be more specifically examined as a contributing factor to compromised/ failed fracture healing.

\section{Acknowledgments}

We thank The University of Queensland's Biological Resource's Herston Medical Research Facility staff, who contributed to animal husbandry, for their excellent services; the Queensland Institute for Medical Research Histology Service for tissue processing; and Elise Farrington for providing some experimental assistance.

\section{Supplemental Data}

Supplemental material for this article can be found at http://dx.doi.org/10.1016/j.ajpath.2014.08.017.

\section{References}

1. Andersson G; American Academy of Orthopaedic Surgeons: The Burden of Musculoskeletal Diseases in the United States: Prevalence, Societal, and Economic Cost. Rosemont, IL, American Academy of Orthopaedic Surgeons, 2008. pp ix, 247

2. Hernlund E, Svedbom A, Ivergard M, Compston J, Cooper C Stenmark J, McCloskey EV, Jonsson B, Kanis JA: Osteoporosis in the European Union: medical management, epidemiology and economic burden: a report prepared in collaboration with the International Osteoporosis Foundation (IOF) and the European Federation of Pharmaceutical Industry Associations (EFPIA). Arch Osteoporos 2013, 8:1-115

3. Heckman JD, Sarasohn-Kahn J: The economics of treating tibia fractures: the cost of delayed unions. Bull Hosp Jt Dis 1997, 56: 63-72

4. Claes L, Recknagel S, Ignatius A: Fracture healing under healthy and inflammatory conditions. Nat Rev Rheumatol 2012, 8:133-143

5. Schindeler A, McDonald MM, Bokko P, Little DG: Bone remodeling during fracture repair: the cellular picture. Semin Cell Dev Biol 2008, 19:459-466

6. Dimmen S, Nordsletten L, Madsen JE: Parecoxib and indomethacin delay early fracture healing: a study in rats. Clin Orthop Relat Res 2009, 467:1992-1999

7. Schmidt-Bleek K, Schell H, Schulz N, Hoff P, Perka C, Buttgereit F, Volk HD, Lienau J, Duda GN: Inflammatory phase of bone healing initiates the regenerative healing cascade. Cell Tissue Res 2012, 347: $567-573$

8. Street J, Bao M, deGuzman L, Bunting S, Peale FV Jr, Ferrara N, Steinmetz H, Hoeffel J, Cleland JL, Daugherty A, van Bruggen N, Redmond HP, Carano RA, Filvaroff EH: Vascular endothelial growth factor stimulates bone repair by promoting angiogenesis and bone turnover. Proc Natl Acad Sci U S A 2002, 99:9656-9661

9. Bourque WT, Gross M, Hall BK: Expression of four growth factors during fracture repair. Int J Dev Biol 1993, 37:573-579

10. Einhorn TA: The cell and molecular biology of fracture healing. Clin Orthop Relat Res 1998, (355 Suppl):S7-S21

11. Gerstenfeld LC, Cullinane DM, Barnes GL, Graves DT, Einhorn TA: Fracture healing as a post-natal developmental process: molecular, 
spatial, and temporal aspects of its regulation. J Cell Biochem 2003, $88: 873-884$

12. Andrew JG, Andrew SM, Freemont AJ, Marsh DR: Inflammatory cells in normal human fracture healing. Acta Orthop Scand 1994, 65: 462-466

13. Oni OO: The early stages of the repair of adult human diaphyseal fractures. Injury 1997, 28:521-525

14. Alexander KA, Chang MK, Maylin ER, Kohler T, Muller R, Wu AC, Van Rooijen N, Sweet MJ, Hume DA, Raggatt LJ, Pettit AR: Osteal macrophages promote in vivo intramembranous bone healing in a mouse tibial injury model. J Bone Miner Res 2011, 26:1517-1532

15. Xing Z, Lu C, Hu D, Yu YY, Wang X, Colnot C, Nakamura M, Wu Y, Miclau T, Marcucio RS: Multiple roles for CCR2 during fracture healing. Dis Model Mech 2010, 3:451-458

16. Hankemeier S, Grassel S, Plenz G, Spiegel HU, Bruckner P, Probst A: Alteration of fracture stability influences chondrogenesis, osteogenesis and immigration of macrophages. J Orthop Res 2001, 19:531-538

17. Wang X, Yu YY, Lieu S, Yang F, Lang J, Lu C, Werb Z, Hu D, Miclau T, Marcucio R, Colnot C: MMP9 regulates the cellular response to inflammation after skeletal injury. Bone 2013, 52: $111-119$

18. Heiner DE, Meyer MH, Frick SL, Kellam JF, Fiechtl J, Meyer RA Jr: Gene expression during fracture healing in rats comparing intramedullary fixation to plate fixation by DNA microarray. J Orthop Trauma 2006, 20:27-38

19. Gerstenfeld LC, Cho TJ, Kon T, Aizawa T, Tsay A, Fitch J, Barnes GL, Graves DT, Einhorn TA: Impaired fracture healing in the absence of TNF-alpha signaling: the role of TNF-alpha in endochondral cartilage resorption. J Bone Miner Res 2003, 18:1584-1592

20. Glass GE, Chan JK, Freidin A, Feldmann M, Horwood NJ, Nanchahal J: TNF-alpha promotes fracture repair by augmenting the recruitment and differentiation of muscle-derived stromal cells. Proc Natl Acad Sci U S A 2011, 108:1585-1590

21. Wynn TA, Chawla A, Pollard JW: Macrophage biology in development, homeostasis and disease. Nature 2013, 496:445-455

22. Schulz C, Gomez Perdiguero E, Chorro L, Szabo-Rogers H, Cagnard N, Kierdorf K, Prinz M, Wu B, Jacobsen SE, Pollard JW, Frampton J, Liu KJ, Geissmann F: A lineage of myeloid cells independent of Myb and hematopoietic stem cells. Science 2012, 336: $86-90$

23. Hashimoto D, Chow A, Noizat C, Teo P, Beasley MB, Leboeuf M, Becker CD, See P, Price J, Lucas D, Greter M, Mortha A, Boyer SW, Forsberg EC, Tanaka M, van Rooijen N, Garcia-Sastre A, Stanley ER, Ginhoux F, Frenette PS, Merad M: Tissue-resident macrophages self-maintain locally throughout adult life with minimal contribution from circulating monocytes. Immunity 2013, 38: 792-804

24. Yona S, Kim KW, Wolf Y, Mildner A, Varol D, Breker M, StraussAyali D, Viukov S, Guilliams M, Misharin A, Hume DA, Perlman H, Malissen B, Zelzer E, Jung S: Fate mapping reveals origins and dynamics of monocytes and tissue macrophages under homeostasis. Immunity 2013, 38:79-91

25. Shi C, Pamer EG: Monocyte recruitment during infection and inflammation. Nat Rev Immunol 2011, 11:762-774

26. Parihar A, Eubank TD, Doseff AI: Monocytes and macrophages regulate immunity through dynamic networks of survival and cell death. J Innate Immun 2010, 2:204-215

27. Martinez FO, Sica A, Mantovani A, Locati M: Macrophage activation and polarization. Front Biosci 2008, 13:453-461

28. Chang MK, Raggatt LJ, Alexander KA, Kuliwaba JS, Fazzalari NL, Schroder K, Maylin ER, Ripoll VM, Hume DA, Pettit AR: Osteal tissue macrophages are intercalated throughout human and mouse bone lining tissues and regulate osteoblast function in vitro and in vivo. J Immunol 2008, 181:1232-1244

29. Winkler IG, Sims NA, Pettit AR, Barbier V, Nowlan B, Helwani F, Poulton IJ, van Rooijen N, Alexander KA, Raggatt LJ, Levesque JP:
Bone marrow macrophages maintain hematopoietic stem cell (HSC) niches and their depletion mobilizes HSCs. Blood 2010, 116: 4815-4828

30. Cho SW, Soki FN, Koh AJ, Eber MR, Entezami P, Park SI, van Rooijen N, McCauley LK: Osteal macrophages support physiologic skeletal remodeling and anabolic actions of parathyroid hormone in bone. Proc Natl Acad Sci U S A 2014, 111:1545-1550

31. Guihard P, Danger Y, Brounais B, David E, Brion R, Delecrin J, Richards CD, Chevalier S, Redini F, Heymann D, Gascan H, Blanchard F: Induction of osteogenesis in mesenchymal stem cells by activated monocytes/macrophages depends on oncostatin $\mathrm{M}$ signaling. Stem Cells 2012, 30:762-772

32. Nicolaidou V, Wong MM, Redpath AN, Ersek A, Baban DF, Williams LM, Cope AP, Horwood NJ: Monocytes induce STAT3 activation in human mesenchymal stem cells to promote osteoblast formation. PLoS One 2012, 7:e39871

33. Lyons FG, Al-Munajjed AA, Kieran SM, Toner ME, Murphy CM, Duffy GP, O'Brien FJ: The healing of bony defects by cell-free collagen-based scaffolds compared to stem cell-seeded tissue engineered constructs. Biomaterials 2010, 31:9232-9243

34. Chen Z, Wu C, Gu W, Klein T, Crawford R, Xiao Y: Osteogenic differentiation of bone marrow MSCs by beta-tricalcium phosphate stimulating macrophages via BMP2 signalling pathway. Biomaterials 2014, 35:1507-1518

35. Matthys R, Perren SM: Internal fixator for use in the mouse. Injury 2009, 40(Suppl 4):S103-S109

36. Ueno M, Uchida K, Takaso M, Minehara H, Suto K, Takahira N, Steck R, Schuetz MA, Itoman M: Distribution of bone marrow-derived cells in the fracture callus during plate fixation in a green fluorescent protein-chimeric mouse model. Exp Anim 2011, 60:455-462

37. Steck R, Ueno M, Gregory L, Rijken N, Wullschleger ME, Itoman M, Schuetz MA: Influence of internal fixator flexibility on murine fracture healing as characterized by mechanical testing and microCT imaging. J Orthop Res 2011, 29:1245-1250

38. Burnett SH, Kershen EJ, Zhang J, Zeng L, Straley SC, Kaplan AM, Cohen DA: Conditional macrophage ablation in transgenic mice expressing a Fas-based suicide gene. J Leukoc Biol 2004, 75: 612-623

39. Hume DA, Pavli P, Donahue RE, Fidler IJ: The effect of human recombinant macrophage colony-stimulating factor (CSF-1) on the murine mononuclear phagocyte system in vivo. J Immunol 1988, 141:3405-3409

40. Hume DA: The mononuclear phagocyte system. Curr Opin Immunol 2006, 18:49-53

41. Sasmono RT, Oceandy D, Pollard JW, Tong W, Pavli P, Wainwright BJ, Ostrowski MC, Himes SR, Hume DA: A macrophage colony-stimulating factor receptor-green fluorescent protein transgene is expressed throughout the mononuclear phagocyte system of the mouse. Blood 2003, 101:1155-1163

42. Grongroft I, Heil P, Matthys R, Lezuo P, Tami A, Perren S, Montavon P, Ito K: Fixation compliance in a mouse osteotomy model induces two different processes of bone healing but does not lead to delayed union. J Biomech 2009, 42:2089-2096

43. Ueno M, Urabe K, Naruse K, Uchida K, Minehara H, Yamamoto T, Steck R, Gregory L, Wullschleger ME, Schuetz MA, Itoman M: Influence of internal fixator stiffness on murine fracture healing: two types of fracture healing lead to two distinct cellular events and FGF-2 expressions. Exp Anim 2011, 60:79-87

44. Wu CA, Pettit AR, Toulson S, Grondahl L, Mackie EJ, Cassady AI: Responses in vivo to purified poly(3-hydroxybutyrate-co-3hydroxyvalerate) implanted in a murine tibial defect model. J Biomed Mater Res A 2009, 91:845-854

45. Cherayil BJ, Chaitovitz S, Wong C, Pillai S: Molecular cloning of a human macrophage lectin specific for galactose. Proc Natl Acad Sci U S A 1990, 87:7324-7328

46. Henderson NC, Sethi T: The regulation of inflammation by galectin3. Immunol Rev 2009, 230:160-171 
47. Sato S, Hughes RC: Regulation of secretion and surface expression of Mac-2, a galactoside-binding protein of macrophages. J Biol Chem 1994, 269:4424-4430

48. Colnot C, Sidhu SS, Balmain N, Poirier F: Uncoupling of chondrocyte death and vascular invasion in mouse galectin 3 null mutant bones. Dev Biol 2001, 229:203-214

49. Ortega N, Behonick DJ, Colnot C, Cooper DN, Werb Z: Galectin-3 is a downstream regulator of matrix metalloproteinase- 9 function during endochondral bone formation. Mol Biol Cell 2005, 16:3028-3039

50. Peng H, Usas A, Olshanski A, Ho AM, Gearhart B, Cooper GM, Huard J: VEGF improves, whereas sFlt1 inhibits, BMP2-induced bone formation and bone healing through modulation of angiogenesis. J Bone Miner Res 2005, 20:2017-2027

51. Hume DA, Macdonald KP: Therapeutic applications of macrophage colony-stimulating factor (CSF-1) and antagonists of CSF-1 receptor (CSF-1R) signaling. Blood 2012, 119:1810-1820

52. Kon T, Cho TJ, Aizawa T, Yamazaki M, Nooh N, Graves D, Gerstenfeld LC, Einhorn TA: Expression of osteoprotegerin, receptor activator of NF-kappaB ligand (osteoprotegerin ligand) and related proinflammatory cytokines during fracture healing. J Bone Miner Res 2001, 16:1004-1014

53. Blumer MJ, Longato S, Fritsch H: Localization of tartrate-resistant acid phosphatase (TRAP), membrane type-1 matrix metalloproteinases (MT1-MMP) and macrophages during early endochondral bone formation. J Anat 2008, 213:431-441

54. McDonald MM, Morse A, Mikulec K, Peacock L, Baldock PA, Kostenuik PJ, Little DG: Matrix metalloproteinase-driven endochondral fracture union proceeds independently of osteoclast activity. J Bone Miner Res 2013, 28:1550-1560

55. Blumer MJ, Longato S, Fritsch H: Structure, formation and role of cartilage canals in the developing bone. Ann Anat 2008, 190:305-315

56. Lucas T, Waisman A, Ranjan R, Roes J, Krieg T, Muller W, Roers A, Eming SA: Differential roles of macrophages in diverse phases of skin repair. J Immunol 2010, 184:3964-3977

57. Ramachandran P, Pellicoro A, Vernon MA, Boulter L, Aucott RL, Ali A, Hartland SN, Snowdon VK, Cappon A, Gordon-Walker TT, Williams MJ, Dunbar DR, Manning JR, van Rooijen N, Fallowfield JA, Forbes SJ, Iredale JP: Differential Ly-6C expression identifies the recruited macrophage phenotype, which orchestrates the regression of murine liver fibrosis. Proc Natl Acad Sci U S A 2012, 109: E3186-E3195

58. Sica A, Mantovani A: Macrophage plasticity and polarization: in vivo veritas. J Clin Invest 2012, 122:787-795

59. Adutler-Lieber S, Ben-Mordechai T, Naftali-Shani N, Asher E, Loberman D, Raanani E, Leor J: Human macrophage regulation via interaction with cardiac adipose tissue-derived mesenchymal stromal cells. J Cardiovasc Pharmacol Ther 2013, 18:78-86

60. Dreier R, Wallace S, Fuchs S, Bruckner P, Grassel S: Paracrine interactions of chondrocytes and macrophages in cartilage degradation: articular chondrocytes provide factors that activate macrophagederived pro-gelatinase B (pro-MMP-9). J Cell Sci 2001, 114: $3813-3822$

61. Fahy N, Lehmann J, de Vries-van Melle M, Grotenhuis N, Farrell E, Murphy JM, Bastiaansen-Jenniskens Y, van Osch GJVM: M1 polarised macrophages are potential mediators of the antichondrogenic effects of osteoarthritis synovium. Eur Cells Mater 2013, 26:27

62. Rifas L, Shen V, Mitchell K, Peck WA: Macrophage-derived growth factor for osteoblast-like cells and chondrocytes. Proc Natl Acad Sci U S A 1984, 81:4558-4562

63. Sasmono RT, Ehrnsperger A, Cronau SL, Ravasi T, Kandane R, Hickey MJ, Cook AD, Himes SR, Hamilton JA, Hume DA: Mouse neutrophilic granulocytes express mRNA encoding the macrophage colony-stimulating factor receptor (CSF-1R) as well as many other macrophage-specific transcripts and can transdifferentiate into macrophages in vitro in response to CSF-1. J Leukoc Biol 2007, 82: $111-123$

64. MacDonald KP, Rowe V, Bofinger HM, Thomas R, Sasmono T, Hume DA, Hill GR: The colony-stimulating factor 1 receptor is expressed on dendritic cells during differentiation and regulates their expansion. J Immunol 2005, 175:1399-1405

65. McDonald MM, Dulai S, Godfrey C, Amanat N, Sztynda T, Little DG: Bolus or weekly zoledronic acid administration does not delay endochondral fracture repair but weekly dosing enhances delays in hard callus remodeling. Bone 2008, 43:653-662

66. McDonald MM, Morse A, Peacock L, Mikulec K, Schindeler A, Little DG: Characterization of the bone phenotype and fracture repair in osteopetrotic incisors absent rats. J Orthop Res 2011, 29:726-733

67. Ulrich-Vinther M, Andreassen TT: Osteoprotegerin treatment impairs remodeling and apparent material properties of callus tissue without influencing structural fracture strength. Calcif Tissue Int 2005, 76: 280-286

68. Matos MA, Tannuri U, Guarniero R: The effect of zoledronate during bone healing. J Orthop Traumatol 2010, 11:7-12

69. Gerstenfeld LC, Sacks DJ, Pelis M, Mason ZD, Graves DT, Barrero M, Ominsky MS, Kostenuik PJ, Morgan EF, Einhorn TA: Comparison of effects of the bisphosphonate alendronate versus the RANKL inhibitor denosumab on murine fracture healing. J Bone Miner Res 2009, 24:196-208

70. Lu C, Xing Z, Wang X, Mao J, Marcucio RS, Miclau T: Anti-inflammatory treatment increases angiogenesis during early fracture healing. Arch Orthop Trauma Surg 2012, 132:1205-1213

71. Wu AC, Raggatt LJ, Alexander KA, Pettit AR: Unraveling macrophage contributions to bone repair. Bonekey Rep 2013, 2:373

72. Abou-Khalil R, Yang F, Mortreux M, Lieu S, Yu YY, Wurmser M, Pereira C, Relaix F, Miclau T, Marcucio RS, Colnot C: Delayed bone regeneration is linked to chronic inflammation in murine muscular dystrophy. J Bone Miner Res 2014, 29:304-315 